\title{
Tendencias en el número de médicos titulados anualmente en el Perú, 2007-2016
}

\section{Trends in the number of physicians annually graduating in Peru, 2007-2016}

Correspondencia Alvaro Taype-Rondan Av. La Fontana 550, La Molina, Lima, Perú

Teléfono: (+51) (01) 3171000 alvaro.taype.r@gmail.com

Recibido: 22/01/2019

Arbitrado por pares

Aprobado: 27/02/2019

Citar como: Peralta Cl, GarciaSolorzano F, Zafra-Tanaka JH, Huapaya-Huertas OS, Taype-Rondan

A. Tendencias en el número de médicos titulados anualmente en el Perú, 2007-2016. Acta Med Peru. 2019;36(1):19-25
C. Ichiro Peralta', Franko Garcia-Solorzano², Jessica H. Zafra-Tanaka³, Oscar S. Huapaya-Huertas ${ }^{4}$, Alvaro Taype-Rondan ${ }^{5}$.

1 Sociedad Científica de Estudiantes de Medicina Villarrealinos, Facultad de Medicina Hipólito Unanue, Universidad Nacional Federico Villarreal. Lima, Perú.

2 Universidad Ricardo Palma. Lima, Perú.

3 CRONICAS Centro de Excelencia en Enfermedades Crónicas, Universidad Peruana Cayetano Heredia. Lima, Perú.

4 Escuela de Medicina Humana, Universidad Científica del Sur. Lima, Perú.

5 Unidad de Investigación para la Generación y Síntesis de Evidencias en Salud, Universidad San Ignacio de Loyola. Lima, Perú.

\section{RESUMEN}

Objetivo: Describir la tendencia en el número de médicos que se titularon durante el periodo 2007-2016 en Perú, en forma general y en subgrupos de acuerdo a las características ligadas a la universidad en la que cursaron los estudios de pregrado. Materiales y métodos: Estudio descriptivo. Se obtuvo el listado de todos los médicos colegiados entre 2007-2016 por medio de la página web del Colegio Médico del Perú; mientras que la fecha de titulación y universidad de procedencia provino de la página web de la Superintendencia Nacional de Educación Superior Universitaria (SUNEDU). Para evaluar las tendencias, se utilizó la prueba de correlación de Spearman. Resultados: En el periodo de estudio se colegiaron 27611 médicos a nivel nacional, con una tendencia anual creciente en la cantidad de médicos titulados $(p<0,001)$. Entre los egresados de universidades peruanas, se encontró un incremento del número de médicos que estudiaron en universidades de Lima $(p<0,001)$ y de la región costa $(p<0,001)$. Adicionalmente, se evidenció un incremento en la cantidad de titulados provenientes de universidades privadas de Lima $(p<0,001)$ y de provincias $(p<0,001)$. Conclusiones: El número de médicos titulados aumenta anualmente, con predominio de aquellos provenientes de universidades de Lima, la costa y universidades privadas. Se evidencia la necesidad urgente de políticas que regulen este crecimiento, con la finalidad de evitar problemas de calidad educativa y empleabilidad.

Palabras clave: Educación de pregrado en Medicina; Recursos humanos en salud; Necesidades y demandas de servicios de salud; Médicos; Perú (fuente: DeCS BIREME) 


\section{ABSTRACT}

Objective. To describe the trends in the number of physicians who graduated from 2007 to 2016 in Peru, both in a general manner as well as in subgroups based on the school they studied. Materials and Methods. This is a descriptive and retrospective study. A list of all physicians who graduated during the 2007-2016 period was obtained from the Peruvian College of Physicians website. Their graduating dates and the schools where they studied were obtained from the National University Education Superintendence (SUNEDU, according to its Spanish initials). Trends were assessed using the Spearman correlation test. Results: During the study period, 27,611 physicians graduated in Peru. There was an annual increase in the total number of graduates $(p<0.001)$. Amongst graduates from Peruvian medical schools, there was an increase in the number of physicians who studied in Lima $(p<0.001)$ and in the Peruvian coast $(p<0.001)$. Also, there were a greater number of graduated physicians from Lima $(p<0.001)$ and other provinces medical schools $(p<0.001)$. Conclusions. The number of graduated physicians annually increases, particularly those who studied in Lima, the coast, and private medical schools. We evidence an urgent need for policies for regulating this growth, aiming to avoid employability and educational quality problems.

Keywords: Education, Medical, Undergraduate; Health Manpower; Health Services Needs and Demand; Physicians; Peru (source: MeSH NLM)

\section{INTRODUCCIÓN}

Los médicos juegan un rol fundamental en la gestión y prestación de servicios de salud, por lo que es necesario que cada país cuente con una cantidad adecuada y que las entidades correspondientes aseguren la calidad en la formación de estos recursos humanos ${ }^{[1,2]}$. Si bien el número de profesionales de salud aún es insuficiente en algunas regiones del mundo ${ }^{[3]}$, se ha evidenciado que el $45 \%$ de los países afiliados a la Organización Mundial de la Salud (OMS) presentan una densidad de médicos por 1000 habitantes (hab) menor a uno ${ }^{[4]}$. Asimismo, se ha observado que, en Latinoamérica, el número de escuelas de Medicina está aumentando de forma acelerada ${ }^{[5]}$. Estudios previos han mostrado el incremento de escuelas de Medicina en países como México, en donde el número de escuelas pasó de 10 a 76 entre 1990 y $2000^{[6]}$ o, en Colombia, donde se observó un aumento de 21 a 59 entre los años 1992 y $2011^{[7]}$.

La situación en Perú sigue un curso similar. Así, entre los años 1998 y 2016, se ha evidenciado que el número de escuelas

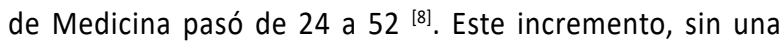
supervisión adecuada, podría haber generado una sobreoferta laboral o una disminución de la calidad educativa. Ante este contexto, en 1999, se intentó regular la formación médica mediante la creación de la Comisión para la Acreditación de Facultades o Escuelas de Medicina Humana (CAFME), la cual desarrolló estándares mínimos para la creación y acreditación de estas instituciones. Sin embargo, unos años después, la función reguladora asignada a la CAFME fue transferida al Consejo Nacional para la Autorización de Universidades, el cual flexibilizó la evaluación y permitió la creación de más escuelas de Medicina ${ }^{[8]}$.

Posteriormente, en el año 2012, se publicó la ley N²9971, impidiendo la creación de nuevas universidades. A pesar de todos estos intentos de regulación, el número de escuelas de Medicina continúa aumentando ${ }^{[8]}$. Por ello, se hace necesaria e importante la realización de un análisis del número de médicos que egresan cada año y las tendencias que se pueden observar. El objetivo de este estudio fue describir la tendencia en el número de médicos que se titularon durante el periodo 2007-2016 en Perú, en forma general y en subgrupos de acuerdo a las características ligadas a la universidad en la que cursaron los estudios de pregrado.

\section{MATERIALES Y MÉTODOS}

\section{Diseño y población de estudio}

Se realizó un estudio descriptivo retrospectivo de análisis de datos secundarios. La población de estudio estuvo conformada por los médicos colegiados en Perú durante el periodo 20072016 registrados en la base de datos del Colegio Médico del Perú (CMP). La muestra fue censal, tomando a la totalidad de los médicos registrados durante dicho periodo.

\section{Variables}

La variable principal fue el año de titulación del médico, la cual fue definida como el año en el cual se emitió el título del médico, según los registros de la Superintendencia Nacional de Educación Superior Universitaria (SUNEDU).

Además, se recolectaron variables de las universidades donde los médicos cursaron sus estudios de pregrado: nombre de la universidad, localización (extranjero, ciudad de Lima, otras ciudades de la región costa, ciudades de la región sierra y ciudades de la región selva) y financiamiento (público o privado). En el caso de universidades con dos o más sedes, como la Universidad de San Martín de Porres (USMP), con sedes en Lima y Chiclayo, se consideró la localización como "Lima" para aquellos que estudiaron en la filial Lima y como "otras ciudades de la costa" para aquellos que estudiaron en Chiclayo.

\section{Procedimientos de recolección de datos}

Para la recolección de datos se siguieron dos pasos: 1) se buscó de manera consecutiva los números de colegiatura correspondientes 
a los médicos colegiados en el periodo 2007-2016 en el servicio "Conoce a tu médico" del CMP (www.cmp.org.pe), de donde se extrajo los nombres y apellidos de los médicos, y 2) los nombres y apellidos de cada médico fueron introducidos en el Registro de Grados y Títulos de la SUNEDU ${ }^{[9]}$, para obtener el año de titulación y la universidad en la cual estudió.

\section{Análisis estadístico}

Para mostrar los resultados en forma descriptiva, se usaron frecuencias relativas y absolutas. Así mismo, se halló la tasa de médicos por 10000 hab. para el Perú y para la ciudad de Lima. Para ello, se dividió el número de médicos por la población del Perú y de la ciudad de Lima según los datos publicados por el Instituto Nacional de Estadística e Informática (INEI) ${ }^{[10]}$. Cabe destacar que, para calcular la población de la ciudad de Lima, se sumó la población de la provincia de Lima más la provincia constitucional del Callao.

Por otro lado, para evaluar la existencia de tendencias se usó la prueba de correlación de Spearman (rho). Se consideró como estadísticamente significativo un valor de $p$ menor de 0,05 . Todos los análisis fueron realizados usando el programa estadístico STATA v.13.0 ${ }^{\circledR}$ (Stata Corp., College Station, TX, US).

\section{Aspectos éticos}

El estudio considera los criterios éticos para las bases de datos de salud y biobancos expuestos en la Declaración de Taipéi de la Asociación Médica Mundial (AMM).

\section{RESULTADOS}

Durante el periodo 2007-2016 se colegiaron 27611 médicos en Perú, de los cuales el $13,7 \%$ se graduaron en universidades del extranjero. La universidad con más médicos titulados fue la USMP, seguida de la Universidad Ricardo Palma (URP). Entre las universidades extranjeras, la que presentó más médicos titulados fue la Escuela Latinoamericana de Medicina de Cuba (40,3\% de todos los médicos que estudiaron en universidades del extranjero) seguida de la Universidad de Aquino de Bolivia (Tabla 1).

Se evidenció una correlación positiva entre el año y el número de titulados (rho=0,939, $p<0,001$ ). La tasa de médicos titulados anualmente por 10000 hab. en el periodo 2007-2016 se incrementó de 0,79 a 1,03 en el Perú, y de 1,08 a 1,27 en Lima (Tabla 2). Asimismo, se encontró una correlación positiva entre el año y el número de titulados de universidades del extranjero ( $r$ ho $=0,842, p=0,002$ ) y de universidades peruanas (rho=0,976, $p<0,001$ ) (Figura 1A).

De los médicos que se graduaron de universidades del extranjero, 1673 (44,2\%) estudiaron en Bolivia y 1615 (42,7\%) en Cuba. Se halló una correlación positiva entre el año y el número de
Tabla 1. Número de médicos titulados en el Perú por universidad, 2007-2016

\begin{tabular}{|c|c|}
\hline Universidades & $\mathbf{N}(\%)$ \\
\hline Perú & $23826(86,3)$ \\
\hline U. de San Martín de Porres & $3050(11,0)$ \\
\hline U. Ricardo Palma & $1821(6,6)$ \\
\hline U. Privada Antenor Orrego & $1761(6,4)$ \\
\hline U. N. Mayor de San Marcos & $1465(5,3)$ \\
\hline U. Privada San Juan Bautista & $1463(5,3)$ \\
\hline U. Católica de Santa María & $1360(4,9)$ \\
\hline U. Peruana Cayetano Heredia & $1112(4,0)$ \\
\hline U. N. Federico Villarreal & $997(3,6)$ \\
\hline U. N. de San Agustín de Arequipa & $965(3,5)$ \\
\hline U. César Vallejo & $920(3,3)$ \\
\hline U. N. de Trujillo & $909(3,3)$ \\
\hline U. Peruana Los Andes & $906(3,3)$ \\
\hline U. N. "San Luis Gonzaga" de Ica & $781(2,8)$ \\
\hline U. San Pedro de Chimbote & $714(2,6)$ \\
\hline U. Científica del Sur & $614(2,2)$ \\
\hline U. N. Pedro Ruiz Gallo & $590(2,1)$ \\
\hline U. N. de San Antonio Abad del Cusco & $496(1,8)$ \\
\hline U. N. del Centro del Perú & $476(1,7)$ \\
\hline U. Privada de Tacna & $454(1,6)$ \\
\hline U. N. de Piura & $410(1,5)$ \\
\hline U. N. José Faustino Sánchez Carrión & $388(1,4)$ \\
\hline U. N. de la Amazonía Peruana & $383(1,4)$ \\
\hline U. N. del Altiplano & $350(1,3)$ \\
\hline U. N. de Cajamarca & $323(1,2)$ \\
\hline U. Particular de Chiclayo & $261(0,9)$ \\
\hline U. N. Jorge Basadre Grohmann & $251(0,9)$ \\
\hline U. N. Hermilio Valdizán & $179(0,6)$ \\
\hline U. N. de Ucayali & $173(0,6)$ \\
\hline U. Peruana de Ciencias Aplicadas & $173(0,6)$ \\
\hline U. Católica Santo Toribio de Mogrovejo & $72(0,3)$ \\
\hline U. Andina Néstor Cáceres Velásquez & $9(0,0)$ \\
\hline Otros países & $3785(13,7)$ \\
\hline Escuela Latinoamericana de Medicina (Cuba) & $1524(5,5)$ \\
\hline U. de Aquino (Bolivia) & $568(2,1)$ \\
\hline U. Privada del Valle (Bolivia) & $444(1,6)$ \\
\hline $\begin{array}{l}\text { U. Mayor Real y Pontificia San Francisco } \\
\text { Xavier de Chuquisaca (Bolivia) }\end{array}$ & $154(0,6)$ \\
\hline U. Mayor de San Andrés (Bolivia) & $119(0,4)$ \\
\hline Otras & $976(3,5)$ \\
\hline
\end{tabular}

U: Universidad; U.N.: Universidad Nacional 
Tabla 2. Número de médicos titulados y tasa de médicos titulados por 10000 habitantes (hab.), por año, en el Perú y Lima

\begin{tabular}{ccccccc} 
Año & $\begin{array}{c}\text { Número de } \\
\text { médicos } \\
\text { titulados en el } \\
\text { Perú }\end{array}$ & $\begin{array}{c}\text { Población } \\
\text { del Perú* }\end{array}$ & $\begin{array}{c}\text { Tasa de } \\
\text { médicos por } \\
10 \text { o00 hab. } \\
\text { en el Perú }\end{array}$ & $\begin{array}{c}\text { Número de } \\
\text { médicos } \\
\text { titulados en la } \\
\text { ciudad Lima }\end{array}$ & $\begin{array}{c}\text { Población } \\
\text { de la ciudad } \\
\text { de Lima* }\end{array}$ & $\begin{array}{c}\text { Tasa de } \\
\text { médicos por } \\
10 \text { 000 hab. } \\
\text { en Lima }\end{array}$ \\
\hline 2007 & 2240 & 28481901 & 0,79 & 946 & 8758889 & 1,08 \\
2008 & 2155 & 28807034 & 0,75 & 920 & 8889774 & 1,03 \\
2009 & 2342 & 29132013 & 0,80 & 966 & 9022535 & 1,07 \\
2010 & 2423 & 29461933 & 0,82 & 940 & 9160384 & 1,03 \\
2011 & 2660 & 29797694 & 0,89 & 1044 & 9303788 & 1,12 \\
2012 & 3092 & 30135875 & 1,03 & 1061 & 9450585 & 1,12 \\
2013 & 2762 & 30475144 & 0,91 & 1054 & 9600114 & 1,10 \\
2014 & 3457 & 30814175 & 1,12 & 1222 & 9751717 & 1,25 \\
2015 & 3229 & 31151643 & 1,04 & 1104 & 9904727 & 1,11 \\
2016 & 3251 & 31488625 & 1,03 & 1273 & 10055200 & 1,27 \\
\hline
\end{tabular}

* Datos obtenidos del Instituto Nacional de Estadística e Informática (población estimada y proyectada por sexo y tasa de crecimiento según años calendarios)

médicos provenientes de Bolivia ( $r h o=0,952, p<0,001$ ) y Cuba ( $r h o=0,697, p<0,001)$. Cabe destacar que, en los médicos provenientes de Cuba, se observaron dos picos: uno en 2012 y otro en el periodo 2014-2015 (Figura 1B).

Con respecto a la región, de los médicos titulados que estudiaron en el Perú, 18206 (76,4\%) estudiaron el pregrado en la costa, $5064(21,3 \%)$ en la sierra y $556(2,3 \%)$ en la selva. Se observó una correlación positiva entre el año y el número de titulados en Lima ( $r h o=0,915, p<0,001)$, en otras ciudades de la costa ( $r h o=0,976, p<0,001$ ) y en ciudades de la selva ( $r h o=0,733$, $p=0,016)$. Se encontró una correlación negativa entre el año y el número de titulados en ciudades de la sierra (rho=-0,673, $p=0,033$ ) (Figura 1C).

De los médicos titulados en el Perú, 14690 (61,7\%), se graduaron en universidades privadas $(29,2 \%$ en Lima y $24,0 \%$ en otras ciudades). Se evidenció una correlación positiva entre el año y el número de titulados en universidades privadas de Lima (rho $=0,927, p<0,001$ ) y de provincia ( $r h o=0,952, p<0,001$ ). Sin embargo, se observó una correlación negativa entre el año y el número de titulados en universidades públicas de Lima (rho=$0,730, p=0,017)$. No se encontró correlación en universidades públicas de provincias $(p=0,292)$ (Figura 1D).

En relación a las tendencias de las cuatro universidades peruanas con mayor número de titulados en el periodo estudiado, se observó una correlación positiva entre el año y el número de médicos titulados de la Universidad Privada Antenor Orrego (UPAO) (rho $=0,976, p<0,001)$ y la Universidad Privada San Juan Bautista (UPSJB) (rho=0,903, $p<0,001)$. No se evidenció correlación entre el año y el número de médicos graduados en la $\operatorname{USMP}(p=0,108)$ y la URP $(p=0,321)$ (Figura $1 \mathrm{E})$.

\section{DISCUSIÓN}

El número de médicos titulados se incrementó durante el periodo 2007-2016, tanto para médicos de universidades peruanas como de universidades del extranjero. Entre los egresados de universidades peruanas, el incremento se dio en universidades de Lima y de otras ciudades de la costa. Se observó un incremento en universidades privadas, pero no en universidades públicas.

Además, en el presente estudio, se encontró que la tasa anual de médicos titulados continúa aumentando, de 0,79 médicos por 10000 hab. en 2007 a 1,03 médicos por 10000 hab. en 2016. No obstante, es importante observar que, en el año 2007, esta tasa ya había superado las estimaciones realizadas en 2008 para Latinoamérica (0,58 médicos por 10000 hab.), Asia (0,60 médicos por 10000 hab.), Norteamérica (0,53 médicos por 10000 hab.) y África (0,17 médicos por 10000 hab. ${ }^{[11]}$.

El aumento en el número de médicos titulados cada año podría tener dos causas principales: el incremento del número de escuelas de Medicina peruanas (ya sea de nuevas universidades privadas o nuevas filiales de estas universidades) y/o el incremento de médicos provenientes de escuelas de Medicina extranjeras. En cuanto al número de escuelas de Medicina peruanas, estas aumentaron de 26 en 2000 a 52 en $2016^{\text {[8] }}$. El crecimiento del número de médicos titulados fue mayor en Lima y en la costa, posiblemente debido a que esta región comprende la mayoría de ciudades con alta densidad demográfica y desarrollo económico. Esta situación podría favorecer la desigual distribución de médicos, la cual ya fue evidenciada por un reporte del Ministerio de Salud en 2017, que menciona que el 43,5\% del total de médicos laboraban en Lima ${ }^{[12]}$. 
A

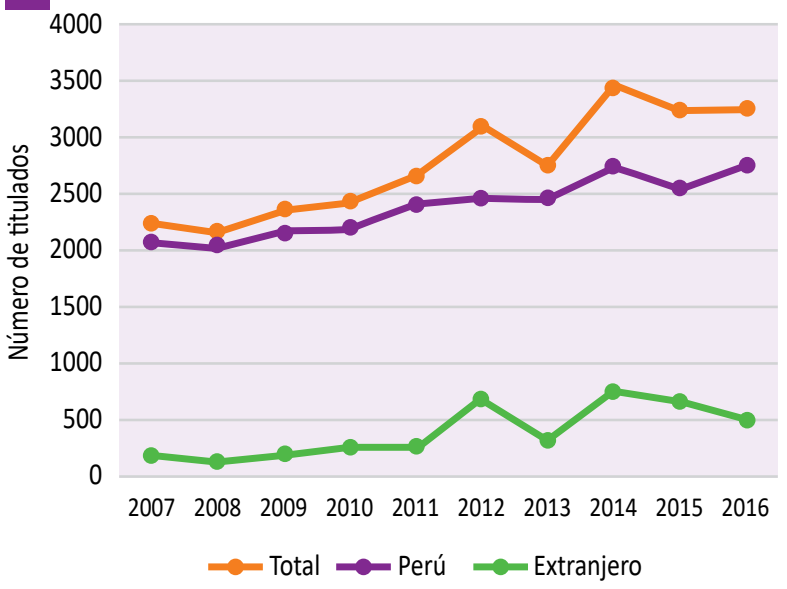

B

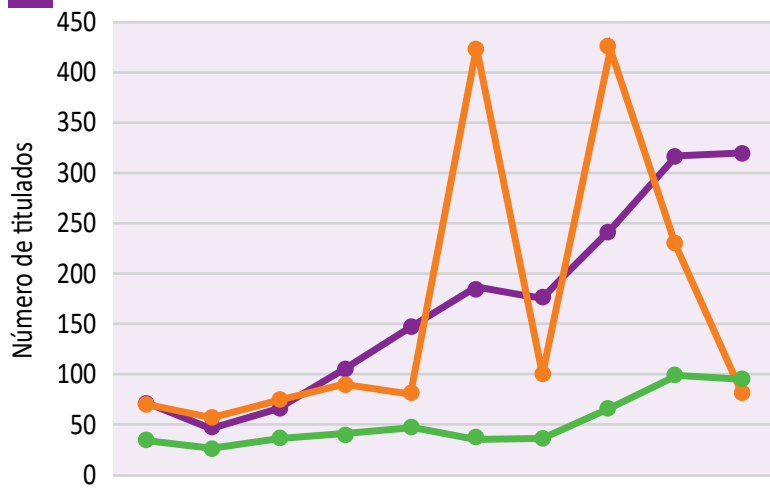

2007200820092010201120122013201420152016 $\rightarrow$ Bolivia $\rightarrow$ Cuba $\rightarrow$ Otros

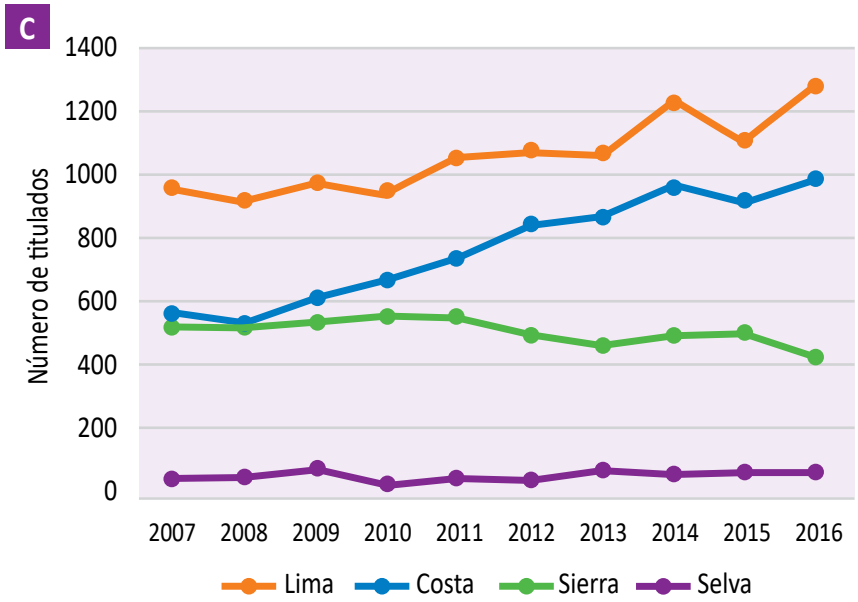

D

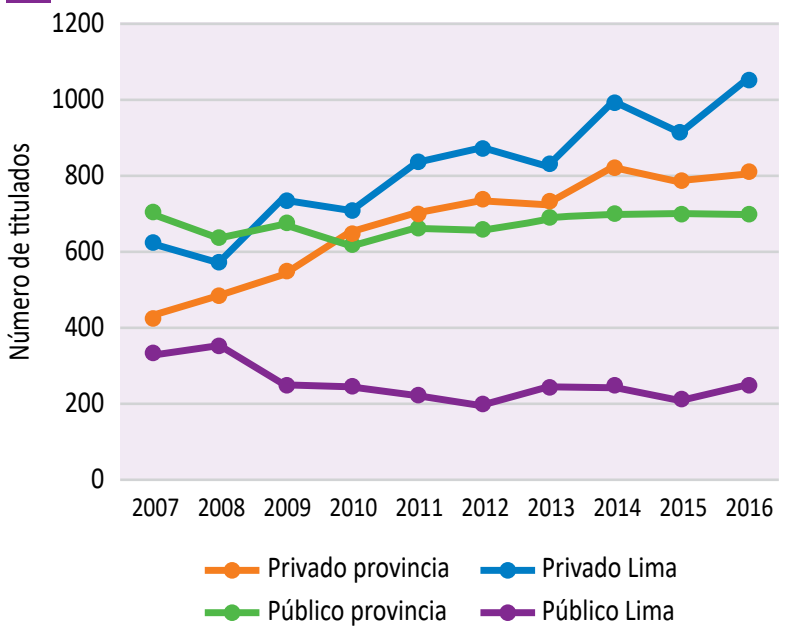

E

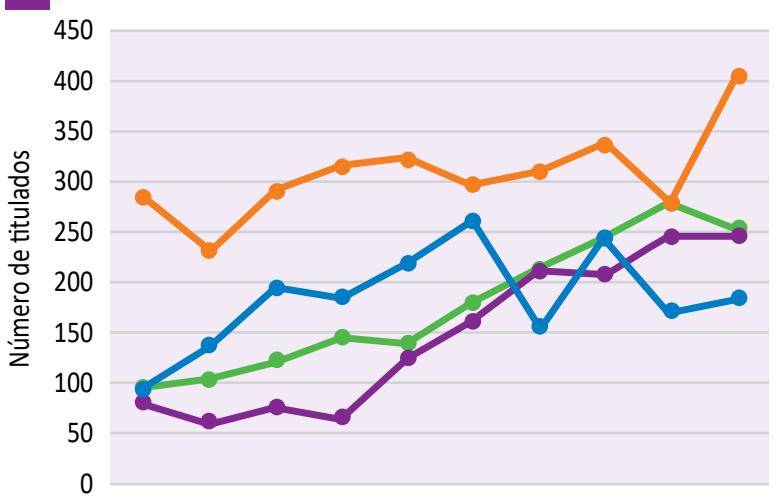

2007200820092010201120122013201420152016

- USMP $\longrightarrow$ URP $\rightleftharpoons$ UPAO UPSJB

USMP: Universidad San Martin de Porres; URP: Universidad Ricardo Palma; UPAO: Universidad Privada Antenor Orrego; UPSJB: Universidad Privada San Juan Bautista.

Figura 1. Tendencias en la titulación de médicos en el Perú (2007-2016). A) Tendencias por financiamiento y ubicación de la universidad. B) Tendencias por países en médicos de universidades extranjeras. C) Tendencias por región en médicos de universidades peruanas. D) Tendencias por tipo de universidad en médicos de universidades peruanas. E) Tendencias de las universidades peruanas con mayor número de médicos titulados. 
Para combatir este centralismo, es necesario regular la distribución de las escuelas de Medicina. Esta falta de regulación lleva a un incremento del número de médicos titulados, lo que puede afectar la calidad de su formación y empleabilidad ${ }^{[13]}$. Con respecto a la calidad de formación, es de esperar que esta se vea dificultada ante la escasa disponibilidad de docentes y campos de práctica clínica ${ }^{[8,14,15]}$. Por ello, es importante asegurar el riguroso cumplimiento de estándares mínimos de calidad (cuya supervisión está actualmente a cargo de la SUNEDU según la Ley $\mathrm{N}^{\circ} 30220$ ), y la divulgación transparente de información al respecto, como los resultados del Examen Nacional de Medicina (ENAM), que no se han hecho públicos desde $2013^{[8]}$.

Mientras que, con respecto a la empleabilidad, se estima que la brecha de necesidad de médicos en el Perú se superará en $2027^{[16]}$. Sin embargo, es posible que esto suceda antes debido al incremento observado del número de médicos titulados anualmente. De forma similar, los médicos suelen concentrarse en ciudades grandes, donde posiblemente ya exista una sobreoferta de estos profesionales ${ }^{[17]}$. El incremento del número de médicos sin una adecuada distribución de la oferta laboral podría estar causando problemas de desempleo, subempleo, multiempleo, bajas remuneraciones ${ }^{[18]}$, insatisfacción laboral y migración al extranjero ${ }^{[19-22]}$.

El número de médicos que han cursado sus estudios en el extranjero también está en aumento, observándose dos picos (2012 y 2014-2015) en la titulación de médicos provenientes de la Escuela Latinoamericana de Medicina de Cuba. Esto se puede atribuir a la entrega de becas a peruanos para estudiar en Cuba desde 2005. El aumento en la cantidad de médicos que estudiaron en el extranjero podría generar heterogeneidad en la formación médica entre países. Por ello, se genera la necesidad de homogenizar ciertas competencias básicas para poder ejercer la Medicina en el Perú. Actualmente, este proceso está a cargo del Colegio Médico del Perú, el cual toma los exámenes de suficiencia profesional obligatorios para la colegiatura de los médicos extranjeros ${ }^{[23]}$. Otra iniciativa actual al respecto es el establecimiento de una nota mínima en el ENAM para poder realizar el Servicio Rural y Urbano Marginal de Salud (Ley $\mathrm{N}^{\circ}$ 23330).

La principal limitación del presente estudio es que solo se ha evaluado el número de médicos titulados, sin evaluar si estos han migrado o han dejado de ejercer la profesión por algún otro motivo. Sin embargo, este estudio es, a nuestro conocimiento, el primer análisis que evalúa la tendencia en el número de médicos titulados en el Perú, utilizando datos de escuelas de Medicina de todo el país, y obteniendo resultados útiles para entender el proceso dinámico de formación médica y poder proponer estrategias de regulación al respecto.

En conclusión, se encontró un incremento del número total de médicos titulados provenientes de universidades peruanas $y$ del extranjero durante el periodo de estudio. Este incremento se evidenció en universidades privadas, pero no en públicas. Los resultados de este estudio evidencian la necesidad urgente de políticas que regulen este crecimiento en las facultades de medicina. De esta forma, se podrían evitar problemas de calidad educativa y empleabilidad de los médicos titulados.

Contribuciones de autoría: ATR y JHZT han participado en la concepción del artículo, la recolección de datos, su redacción y aprobación de la versión final. Además, JHZT realizó el análisis de datos. CIP, FGS y OSHH han participado en la recolección de datos, redacción y aprobación de la versión final.

\section{Fuentes de financiamiento: Autofinanciada.}

Conflictos de interés: Ninguno.

\section{REFERENCIAS BIBLIOGRÁFICAS}

1. Navarro P, Cárdenas Cruz A, Gómez Jiménez FJ. Estudio de los números clausus y graduados en Medicina para su inclusión en un modelo de planificación de necesidades de formación de especialistas médicos en Andalucía. Actual Medica. 2016;(799):154-9.

2. Pierdant-Pérez $G$, Grimaldo-Avilés JI. La discrepancia entre la apertura de nuevas escuelas de medicina en México y la planeación de recursos humanos en salud. Investigacion Educ Medica. 2013;2(6):82-6.

3. World Health Organization (WHO). A universal truth: No health without a workforce [Internet]. Ginebra: WHO; 2014 [citado 04 nov 2018]. Disponible en: http://www.who.int/workforcealliance/ knowledge/resources/GHWA-a_universal_truth_report.pdf

4. World Health Organization (WHO). Global Health Observatory (GHO) data: Density of physicians [Internet]. Ginebra: WHO; 2018 [citado 04 nov 2018]. Disponible en: http://www.who.int/gho/ health_workforce/physicians_density/en/

5. Goic A. Proliferación de escuelas de medicina en Latinoamérica: causas y consecuencias. Rev Med Chil. 2002;130(8):917-24.

6. De la Garza-Aguilar J. Reflexiones sobre la calidad de la carrera de medicina en México. Gac Med Mex. 2005;141(2):129-41.

7. Rozo Uribe RH, Escobar Gaviria RH. La educación médica en Colombia. Revista Med. 2011;19(1):7-9.

8. Mayta-Tristán P, Cuentas M, Núñez-Vergara M. Responsabilidad de las instituciones ante la proliferación de escuelas de medicina en el Perú. Acta Med Peru. 2016;33(3):178-82.

9. Superintendencia Nacional de Educación Superior Universitaria (SUNEDU). Registro de grados y títulos 2018 [Internet]. Lima, Perú: SUNEDU; c2016 [citado 04 nov 2018]. Disponible en: https://www. sunedu.gob.pe/grados-y-titulos/registro-de-grados-y-titulos/

10. Instituto Nacional de Estadística e Informática (INEI). Población y vivienda: Estimaciones y proyecciones de población [Internet]. Lima, Perú: INEI; 2018 [citado 04 nov 2018]. Disponible en: https:// www.inei.gob.pe/estadisticas/indice-tematico/poblacion-yvivienda/

11. Frenk J, Chen L, Bhutta ZA, Cohen J, Crisp N, Evans T, et al. Health professionals for a new century: transforming education to strengthen health systems in an interdependent world. Lancet. 2010;376(9756):1923-58.

12. Dirección General de Personal de la Salud, Ministerio de Salud. Registro Nacional del Personal de la Salud. Información de Recursos Humanos en Salud. Lima, Perú: MINSA; 2016 [citado 04 nov 2018]. Disponible en: http://bvs.minsa.gob.pe/local/MINSA/4041.pdf 
13. World Health Organization (WHO). The 2017 update, Global Health Workforce Statistics [Internet]. Ginebra: WHO; 2017 [citado 04 nov 2018]. Disponible en: http://www.who.int/hrh/statistics/ hwfstats/en/

14. Alva J, Verastegui G, Velasquez E, Pastor R, Moscoso B. Oferta y demanda de campos de práctica clínica para la formación de pregrado de estudiantes de ciencias de la salud en el Perú, 20052009. Rev Peru Med Exp Salud Publica. 2011;28(2):194-201.

15. Montenegro-Idrogo JJ, Montañez-Valverde R, Sánchez-Tonohuye J. Sobredemanda del campo clínico para estudiantes de medicina. Rev Peru Med Exp Salud Publica. 2012;29(1):155-7.

16. Huayanay $C$, Jimenez M, Mantilla E, Mego M, Vermeersch CMJ. Analysis of the health care labor market in Peru (English) [Internet]. Health, Nutrition and Population (HNP) discussion paper. Washington, DC: The International Bank; 2015 [citado 04 nov 2018]. Disponieble en: http:// documents.worldbank.org/curated/en/589771468285030626/ pdf/951150WP0OPUBL00Study0Peru00PUBLICO.pdf

17. Jimenez MM, Bui AL, Mantilla E, Miranda JJ. Human resources for health in Peru: recent trends (2007-2013) in the labour market for physicians, nurses and midwives. Hum Resour Health. 2017;15(1):69.
18. Carrasco Cortez V, Lozano Salazar E, Velásquez Pancca E. Análisis actual y prospectivo de la oferta y demanda de médicos en el Perú 2005-2011. Acta Med Peru. 2008;25(1):22-9.

19. Astor A, Akhtar T, Matallana MA, Muthuswamy V, Olowu FA, Tallo $\mathrm{V}$, et al. Physician migration: views from professionals in Colombia, Nigeria, India, Pakistan and the Philippines. Soc Sci Med. 2005;61(12):2492-500.

20. Pereyra-Elías R, Cassana A, Mezones-Holguín E, Mayta-Tristán P. Diferencias según género en la intención de emigración en médicos recién egresados. An Fac Med. 2013;74(3):211-6.

21. Mayta-Tristán P, Dulanto-Pizzorni A. Prevalencia y factores asociados con la intención de emigración en internos de medicina de una universidad pública, Lima 2007. Rev Peru Med Exp Salud Publica. 2008;25(3):274-8.

22. Instituto Nacional de Estadística e Informática (INEI). Perú: Estadísticas de la emigración internacional de peruanos e inmigración de extranjeros, 1990-2015 [Internet]. Lima, Perú: INEI; 2016 [citado 04 nov 2018]. Disponible en: https://www.inei.gob.pe/media/ MenuRecursivo/publicaciones_digitales/Est/Lib1423/libro.pdf

23. Colegio Médico del Perú. Trámites para el colegiado [Internet]. Lima, Perú: CMP; c2018 [citado 04 nov 2018]. Disponible en: http://cmp. org.pe/tramites-para-el-colegiado/

\title{
Ahora puede enviar sus artículos para Acta Médica Peruana en nuestro Open Journal System:
}

\author{
www.amp.cmp.org.pe
}

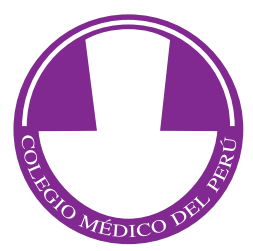

